\title{
Will Your Battery Survive a World With Fast Chargers?
}

\section{Jeremy S. Neubauer and Eric Wood}

National Renewable Energy Laboratory

CITATION: Neubauer, J. and Wood, E., "Will Your Battery Survive a World With Fast Chargers?," SAE Technical Paper 2015-011196, 2015, doi:10.4271/2015-01-1196.

Copyright (C) 2015 SAE International

\begin{abstract}
Fast charging is attractive to battery electric vehicle (BEV) drivers for its ability to enable long-distance travel and to quickly recharge depleted batteries on short notice. However, such aggressive charging and the sustained vehicle operation that results could lead to excessive battery temperatures and degradation. Properly assessing the consequences of fast charging requires accounting for disparate cycling, heating, and aging of individual cells in large BEV packs when subjected to realistic travel patterns, usage of fast chargers, and climates over long durations (i.e., years). The U.S. Department of Energy's Vehicle Technologies Office has supported the National Renewable Energy Laboratory's development of BLAST-V-the Battery Lifetime Analysis and Simulation Tool for Vehicles-to create a tool capable of accounting for all of these factors. We present on the findings of applying this tool to realistic fast charge scenarios. The effects of different travel patterns, climates, battery sizes, battery thermal management systems, and other factors on battery performance and degradation are presented. We find that the impact of realistic fast charging on battery degradation is minimal for most drivers, due to the low frequency of use. However, in the absence of active battery cooling systems, a driver's desired utilization of a BEV and fast charging infrastructure can result in unsafe peak battery temperatures. We find that active battery cooling systems can control peak battery temperatures to safe limits while allowing the desired use of the vehicle.
\end{abstract}

\section{Introduction}

Fast charging is attractive to battery electric vehicle (BEV) drivers for its ability to enable long-distance travel and to quickly recharge depleted batteries on short notice. However, such aggressive charging and the sustained vehicle operation that results could lead to excessive battery temperatures and degradation. Properly assessing the consequences of fast charging requires accounting for disparate cycling, heating, and aging of individual cells in large BEV packs when subjected to realistic travel patterns, usage of fast chargers, and climates over long durations (i.e., years).
In the literature, the impact of high charge rates representative of BEV fast charging on the electrochemical degradation and thermal response of lithium-ion cells has been studied significantly $[\underline{1}, \underline{2}, \underline{3}, \underline{4}$, $\underline{5}, \underline{6}$ ]. However, such studies generally do not treat the thermal effects of operation at the pack level (where cell temperatures can be significantly elevated) and often employ single or repeated full depth-of-discharge fast charge cycles over accelerated test periods that do not address the low frequency of occurrence and partial depthof-discharge cycling expected to occur with BEVs in the real world. Few studies have been published addressing placement strategies for fast chargers. The majority that do exist focus on the interaction of the chargers with the electricity grid [ $\underline{7}-\underline{8}]$, fewer still address optimal placement with respect to vehicle utility []].

The U.S. Department of Energy's Vehicle Technologies Office has supported the National Renewable Energy Laboratory's (NREL's) development of BLAST-V-the Battery Lifetime Analysis and Simulation Tool for Vehicles-to create a tool capable of connecting all of the factors necessary to make a detailed assessment of the battery requirements for and vehicle utility impacts of BEV fast charging in real-world settings. We present the development of the BLAST-V tool and our findings in realistic fast charge scenarios. The effects of different travel patterns, climates, battery sizes, battery thermal management systems (BTMSs), and other factors on battery performance and degradation are presented.

\section{Analysis}

BLAST-V was used to simulate the response of a BEV with and without access to fast charging to assess its impacts on the battery. NREL's BLAST-V is an electric vehicle simulator focused on computing long-term effects of complex operational scenarios on vehicle utility and battery performance. It considers the vehicle powertrain, battery control strategy, driving and charging patterns, local climate, the vehicle-battery-environment thermal system, battery chemistry, and other factors in computing short-term vehicle and battery performance (e.g., vehicle range, battery voltage, state of 


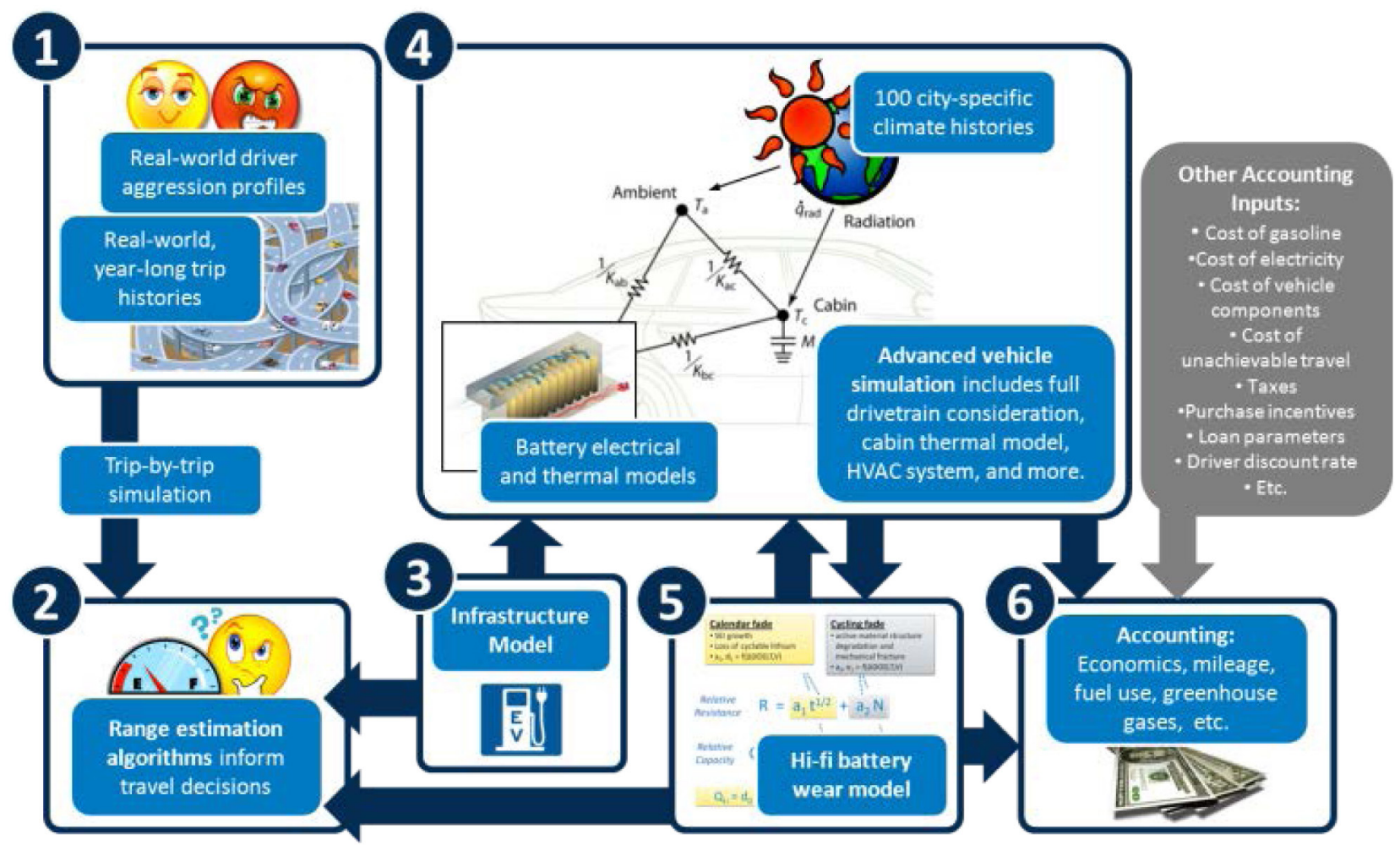

Figure 1. Graphical illustration of BLAST-V simulation algorithms

charge (SOC), and temperature) and long-term vehicle utility and battery degradation. An approximate graphical representation of the key elements and flow of data within BLAST-V is illustrated in Figure 1. Further detail on the methods employed in this simulation can be found in $[\underline{10}-\underline{11}]$.

Prediction of battery degradation in response to arbitrary operating conditions is currently a working area of research in the battery community. Many models that do so are empirically based, interpolating between or extrapolating from different sets of measured battery capacity and resistance data sets $[\underline{12}, \underline{13}, \underline{14}]$. These models require extensively large amounts of data and are often inadequate for extrapolating beyond the duration of the life test data upon which they are based. Alternatively, first-principles models have been proposed, but can be exceedingly complex and limited in scope to a narrow range of operating conditions $[\underline{14}, \underline{15}, \underline{16}, 17]$. NREL has developed a semi-empirical life model that attempts to bridge this gap, offering a combination of increased confidence in interpolations and projections while maintaining simplicity of implementation and a basis in actual laboratory data [18]. BLAST-V incorporates the NREL model for a lithium-ion cell with a nickel-cobalt-aluminum cathode and graphite anode to supply a representative model of battery degradation. Recent updates enable BLAST-V to simulate individual cells within a pack, deploying this degradation model in a highly parallel fashion to investigate heterogeneous cell aging. Another important aspect of BLAST-V is the determination of which trips to take with a BEV and which to forgo. As input driving patterns are generally sourced from real-world operation of conventional vehicles, certain trips (and sequences of trips) will exceed the driving range of the simulated BEV and result in full battery depletion. Given the cost and inconvenience associated with stranded vehicles, BLAST-V assumes BEV drivers will rely on conservative estimates of vehicle range and a detailed knowledge of travel itineraries to avoid running out of charge mid-trip.
BLAST-V structures travel data as a sequence of tours. A tour consists of consecutive trips with the first trip beginning and the last trip ending at the vehicle's home location (with assumed access to charging). Prior to the start of each tour, BLAST-V considers the battery's current SOC, distance and expected duration of pending trips in the tour, historical depletion rates from similar trips, and availability of work/public chargers to estimate battery SOC throughout the potential tour. This estimation informs a go/no-go decision at the beginning of each tour.

If the estimated SOC is maintained above a specified threshold for the entirety of the tour, the simulated driver selects the BEV for travel and the tour is simulated in greater detail considering electrical, thermal, and life models of the battery pack. However, if the SOC is estimated to deplete below the specified threshold, the driver forgoes use of the BEV, and electrical, thermal, and life models of the battery pack are simulated with the vehicle in its parked mode for the duration of the tour. BLAST-V's go/no-go decision for determining $\mathrm{BEV}$ travel is believed to mirror the way that real-world drivers make personal travel decisions. By implementing a low-order planning model prior to tour evaluation, BLAST-V simulates the hundreds of tour decisions a driver makes every year when determining whether their BEV is suitable for a particular tour.

In situations where the estimated battery SOC is not predicted to be maintained above the driver's required threshold, BLAST-V now includes the capability to consider alternate paths of travel and stops at available direct current fast charger (DCFC) stations [19].

If BLAST-V's rerouting algorithm is able to successfully identify a revised travel plan that maintains estimated battery SOC above the driver's minimum requirement, statistics on the rerouted tour are recorded (e.g., number of DCFC stops, duration of DCFC stops, incremental distance relative to original tour) and the rerouted tour is 
simulated in greater detail. However, if an adequate alternate tour is not identified, the driver forgoes use of the BEV, and electrical, thermal, and life models of the battery pack are simulated with the vehicle in its parked mode for the duration of the tour.

For illustrative purposes, consider the details of the tour shown in Figures 2 and $\underline{3}$. Figure 2 shows the tour path consisting of four separate trips for a total of 106 miles. When simulated with a BEV with a 75-mile range (BEV75), the estimated battery SOC falls below a predefined driver tolerance of $15 \%$ midway through the third trip. Because the estimated battery SOC does not stay above the driver's required threshold, BLAST-V considers potential mid-trip stops at DCFC stations for charging. Figure 2 also shows a one-mile buffer around the path of travel (only stations within this buffer are considered), all available DCFC stations in the simulation, and DCFC stations selected for mid-trip charging stops. Travel itineraries and estimated battery SOC for the original tour and the rerouted tour with one DCFC stop are shown in Figure 2. By inserting a 17-minute DCFC stop midway through Trip 3, the estimated battery SOC is maintained above the driver's required threshold for the entire tour.

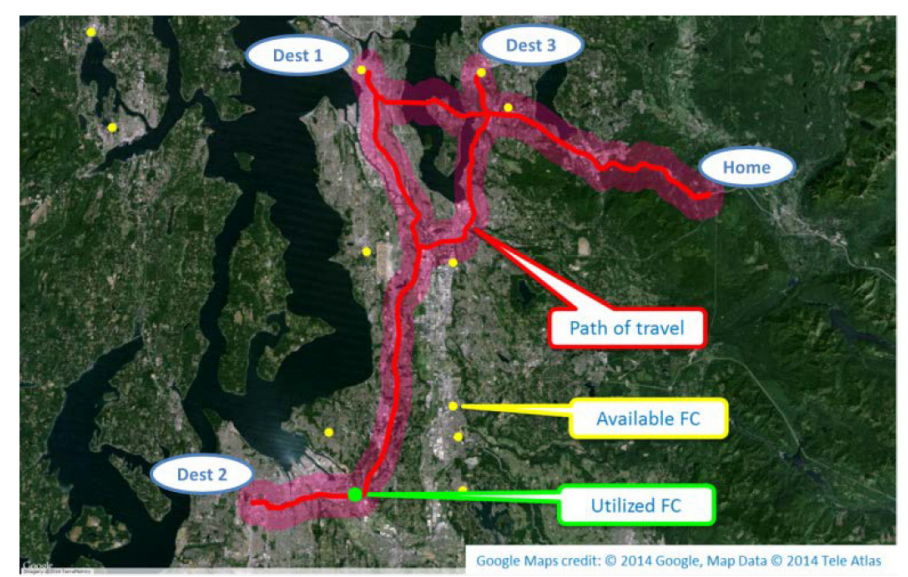

Figure 2. Example tour overlaid with available and utilized DCFC stations
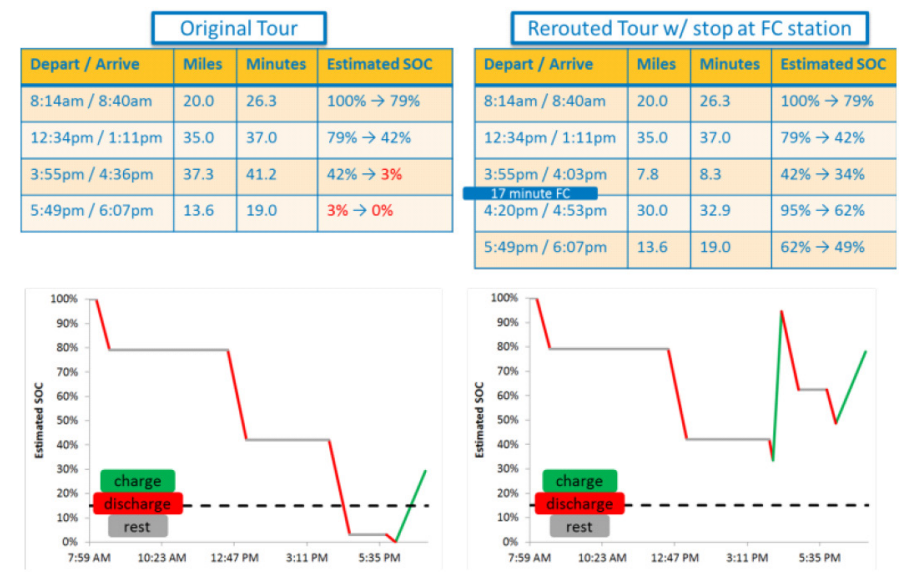

Figure 3. Example original and rerouted travel itineraries and estimated battery SOC

\section{Simulation Parameters}

Historical travel data from the Puget Sound Regional Council's Traffic Choices Study [20] was processed per [10] to yield 317 real-world travel histories, each consisting of 365 continuous days of continuous data. We then filtered these histories to those that accrued 8,000 miles or more over this one-year period for simulation to focus on higher mileage drivers. All 317 histories are plotted in Figure 4 to show the utility factor and the annual mileage they would achieve driving a BEV75 without fast charging. The black points to the upper left of the diagonal line represent the 137 drivers that completed less than 8,000 miles in a conventional vehicle and are therefore excluded from this study. The 91 drivers boxed in the upper right corner of the plot represent those that both completed more than 8,000 miles and achieved a utility factor greater than $80 \%$ in the BEV75. Arguably, these drivers are well suited to such a BEV without fast charging, but are still included. The remaining 89 drivers are high-mileage drivers that achieve low utility factors with a BEV75, and thus drivers that could benefit significantly from range extension methods like fast charging.

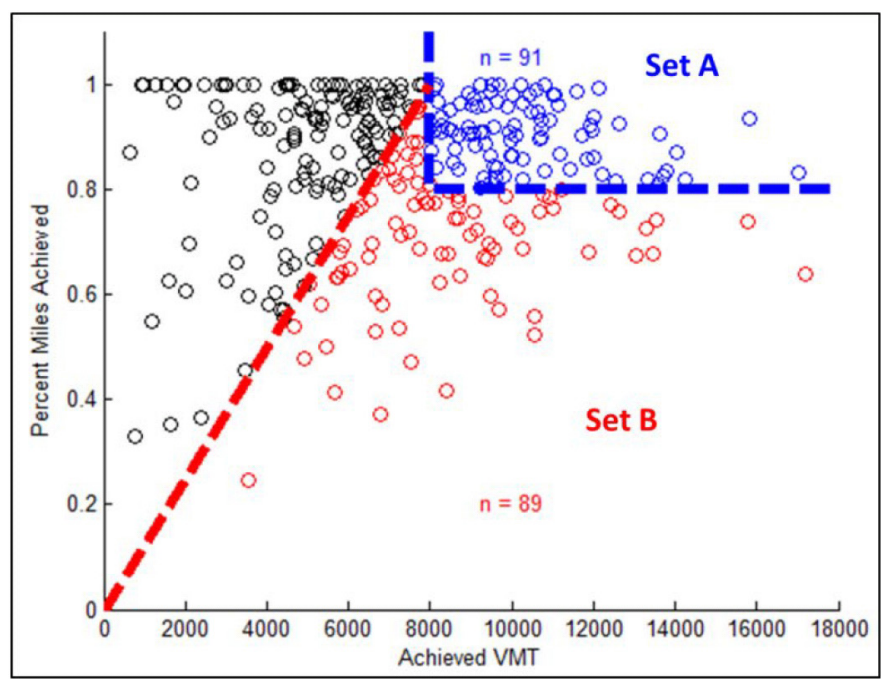

Figure 4. Trip distance, daily distance, annual distance, and trip average speed distributions for all 317 vehicle histories

The effects of two different climates were studied. Seattle, Washington, was selected as it is coincident with the travel data and represents a relatively moderate climate. Phoenix, Arizona, was selected to represent an aggressively hot climate to explore the impact of high environmental temperatures. Typical meteorological year data (consisting of both ambient temperature and solar irradiance) for both locations is taken from [21], and ambient temperatures are illustrated in Figure 5.
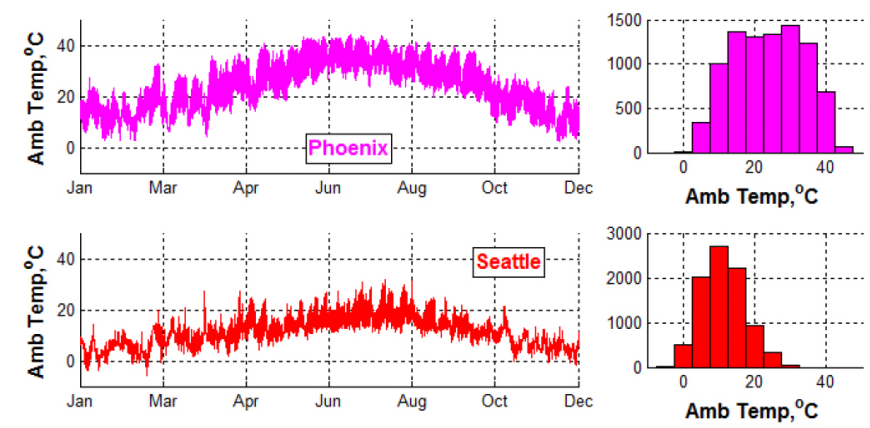

Figure 5. Ambient temperature data for Seattle and Phoenix

We employ a mid-size sedan with technology and performance levels anticipated for a 2020 model year vehicle. We used FASTSim [22] to simulate the vehicle response to the Urban Dynamometer Driving Schedule and Highway Fuel Economy Driving Schedule drive cycles, the results of which are weighted and combined per [23] to approximate the EPA rated range. We further employed FASTSim to 
simulate the vehicle's response to NREL's Drive-Cycle Rapid Investigation, Visualization, and Evaluation (DRIVE) cycle to calculate the vehicle's real-world efficiency [24]. Note that within BLAST-V simulations, auxiliary loads for vehicle heating, ventilating, and air conditioning and BTMS are added separately. Vehicle parameters are given in Table 1.

Table 1. Vehicle Parameters

\begin{tabular}{|l|l|}
\hline $0-60$ mph acceleration & $9 \mathrm{sec}$ \\
\hline $\begin{array}{l}\text { Approximated U.S. Environmental } \\
\text { Protection Agency-rated range }\end{array}$ & $75 \mathrm{miles}$ \\
\hline battery energy & $22.1 \mathrm{kWh}$ \\
\hline motor power & $106 \mathrm{~kW}$ \\
\hline Vehicle curb weight & $1,576 \mathrm{~kg}$ \\
\hline Vehicle efficiency & $220 \mathrm{Wh} / \mathrm{mi}$ on DRIVE cycle \\
\hline
\end{tabular}

Two different battery models were employed in this study, both based on an equivalent circuit model of a lithium-ion battery chemistry employing a nickel-cobalt-aluminum cathode and graphite anode. Further details on the voltage and resistance characteristics of this model are given in [10]. In the first model, a single-cell approximation was used. This effectively assumes that all cells in the pack behave identically with respect to electrical, thermal, and degradation response. We used this model to investigate the difference between three different BTMSs: one with only passive thermal control, one with active cooling when driving, and one with active cooling when driving and charging. Additional details on the cooling system specifications are given in [10].

The second model segregates electrical and degradation responses into 100 individual cells. Initial cell properties are shown in Table 2. Thermal response is treated by creating three cell groupings, assuming temperature is constant across cells within each group, but allowing the temperature of the group to diverge. Thermal properties have been selected to approximately represent the minimum, average, and maximum cell temperatures as observed in field data of a similar BEV [25]. This model will be employed to demonstrate pack behavior, including internal variation, in response to aggressive fast charger use. The same three BTMS options as employed for the single cell model are maintained for this model.

Table 2. Distributed Battery Model Initial Conditions

\begin{tabular}{|l|l|}
\hline Electrical model & 100 discrete nodes \\
\hline BOL capacity variation & $\pm 2 \%$ \\
\hline BOL internal resistance variation & $\pm 5 \%$ \\
\hline Life coefficient variation & $\pm 10 \%$ \\
\hline Thermal model & 3 discrete nodes \\
\hline
\end{tabular}

$\mathrm{BOL}=$ beginning of life

For vehicle charging, we assume a Level 2 charger (6.6 kW AC) is installed at each driver's home and is used in an "opportunity" mode (i.e., whenever the driver is at home, the vehicle is plugged in and charging). A public network of $50 \mathrm{~kW}$ DCFCs is placed as shown in Figure 6, coincident with the 34 DCFCs existing in the state of
Washington as of January 2014 [26]. An additional 306 DCFCs are included outside of the state as well and are available for use in these simulations; however, their utilization is generally low.

The tour planning algorithm discussed in the previous section is employed. A 1-mile radius search zone around the most efficient path of travel is used to identify candidate chargers. A $15 \%$ minimum battery SOC limit is used to address range anxiety effects (i.e., the algorithm will utilize DCFC such that battery SOC stays above 15\%). We further enforce a "depart on time" requirement that maintains the departure time of each trip in a tour. The arrival time at any given location can be delayed by DCFC usage, but only up to the point that it equals the subsequent departure time.

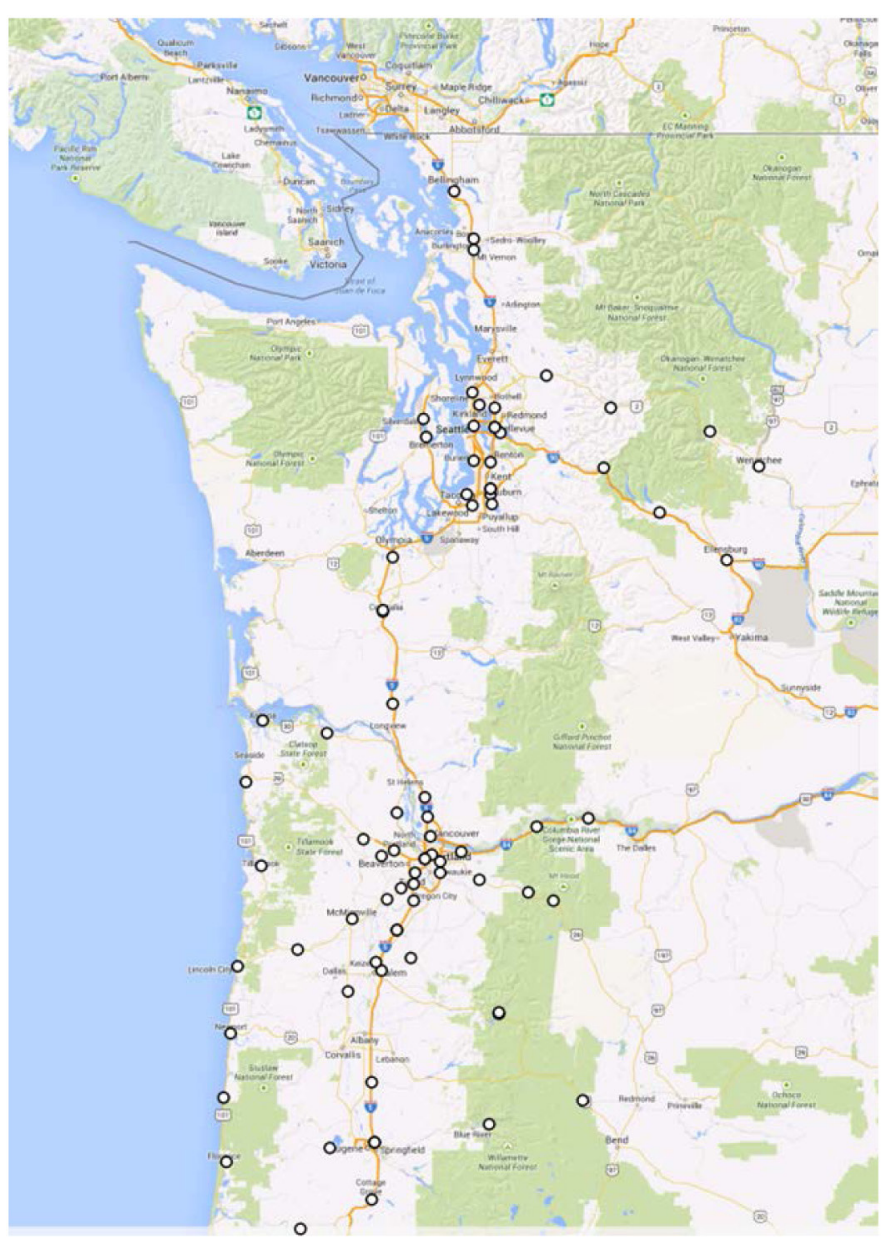

Figure 6. Existing DCFC Stations (source: NREL Alternative Fuels Data Center, January 2014 [26]) (Google Maps credit: (C) 2014 Google, Map Data (C) 2014 Tele Atlas [27])

\section{Baseline Simulations}

Baseline simulations were run using the Seattle climate and a single-cell battery model to check the typical usage of DCFCs resulting from our implemented methodologies and assumptions. Data for these simulations are presented in Figures 7, $\underline{8}, \underline{9}$. These results show that most drivers use fast chargers 10 times per year or less, but extreme cases can reach up to eight times per month. This results in relatively small fractions of total BEV electricity coming from DCFCs, typically less than $10 \%$. When visiting a DCFC, we predict that drivers will arrive with a battery SOC of 18 to $60 \%$ and 
stay between 10 and 22 minutes. Although data on real-world usage of DCFCs are sparse, this appears to agree reasonably well with data reported by the EV Project [28, 29, 30].

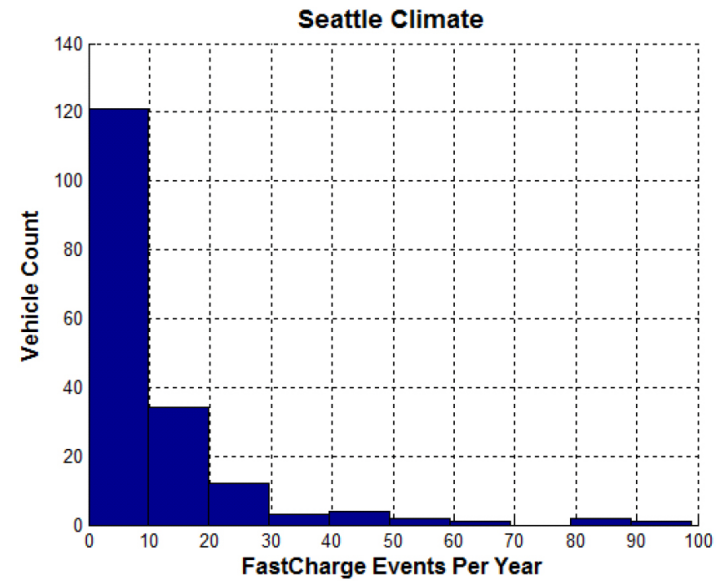

Figure 7. Simulated DCFC usage frequency

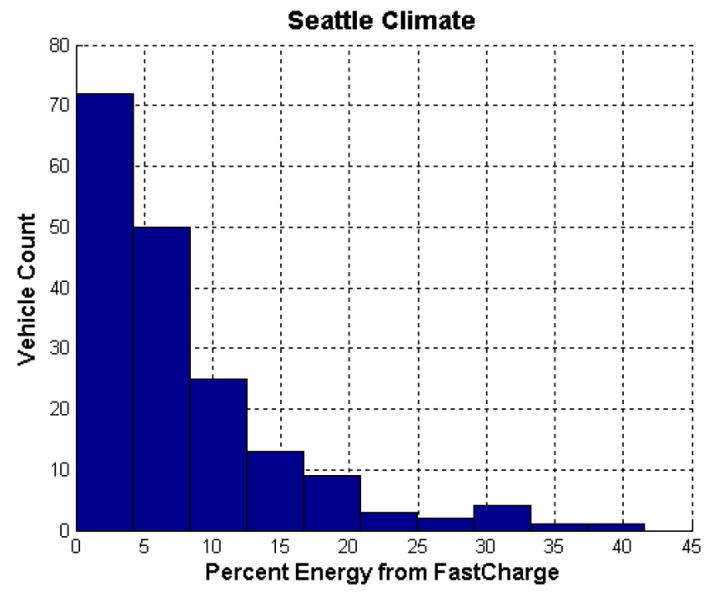

Figure 8. Simulated percent of energy acquired from DCFCs

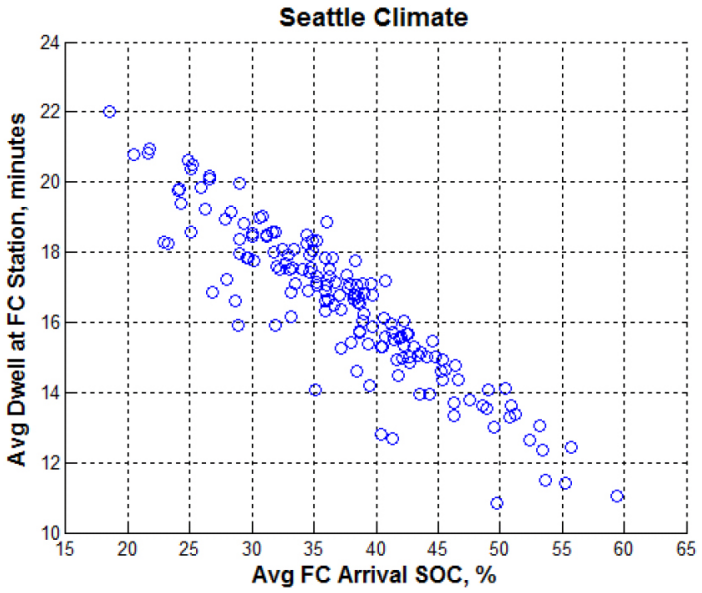

Figure 9. Simulated DCFC dwell time as a function of arrival SOC

\section{Results: Single Node Model}

The effects of three different BTMS in two climates (Seattle and Phoenix) were simulated using our single-cell battery model. For comparison, we simulated cases both with and without the availability of DCFCs. All other variables between simulations are equivalent.
Figure 10 shows the effects of these variables on time-averaged battery temperatures for Seattle and Phoenix. The height of the bars indicates the median of time-averaged battery temperature across all 180 simulated travel histories. The whiskers indicate the minimum and maximum time-averaged battery temperatures across all 180 simulated travel histories. In Seattle, neither the use of DCFCs nor the variation of the BTMSs has any significant effect on timeaveraged battery temperatures for the median driver. For the most extreme travel history that results in the largest time-averaged battery temperature, the addition of DCFC can increase time-averaged battery temperature by $2^{\circ} \mathrm{C}-3^{\circ} \mathrm{C}$ in the presence of a passive BTMS. Where an active BTMS is present, this effect is largely unnoticeable.

Similar trends in time-averaged battery temperature are seen in the Phoenix data when comparing the absence and presence of DCFCs. However, the difference between BTMSs is much more apparent: the presence of active cooling while driving and the additional use of that system in standby mode at a charger noticeably reduce the average battery temperature.
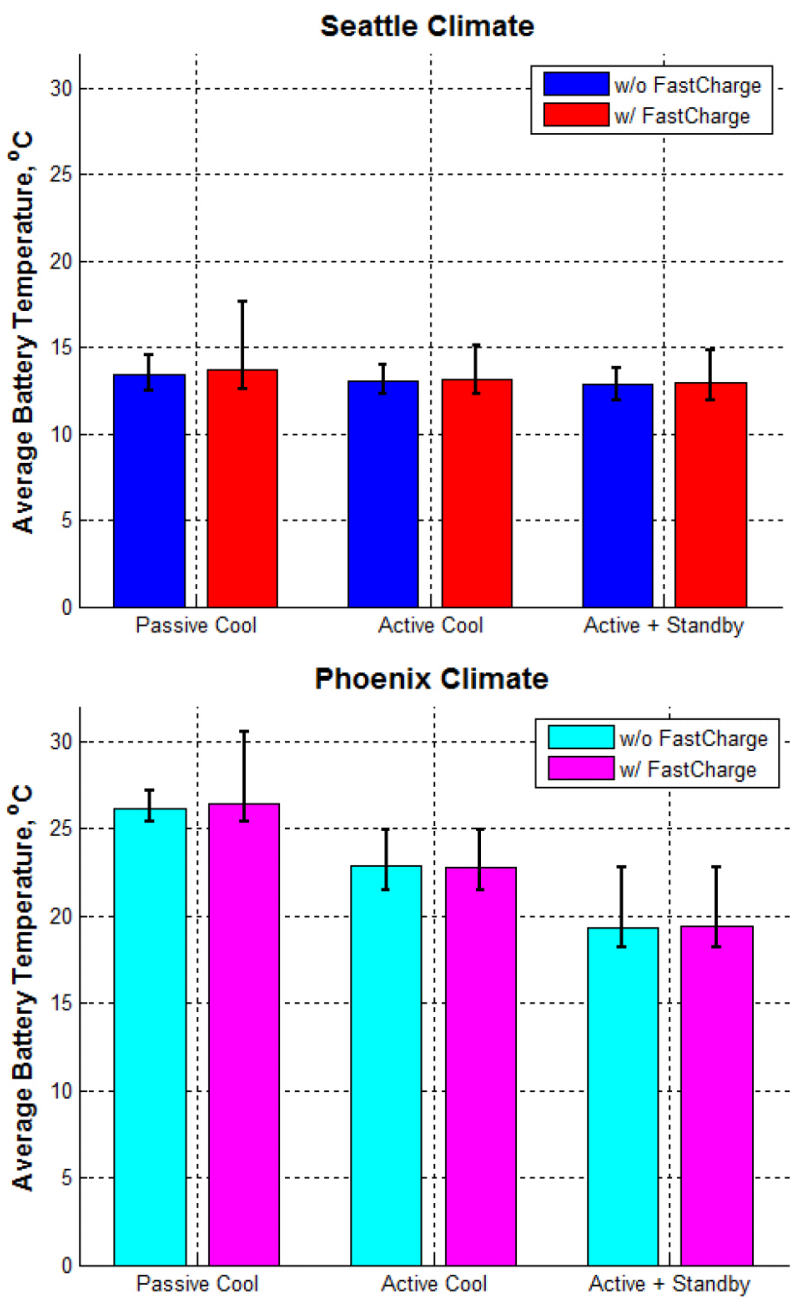

Figure 10. Effect of DCFCs and BTMSs on average battery temperature in Seattle (top) and Phoenix (bottom)

As in our past studies that have shown that BEV battery life is often dependent on calendar fade mechanisms rather than cycling fade mechanisms [10-11], we find that these trends in time-averaged battery temperatures translate almost directly to battery capacity loss. 
As shown in Figure 11, we see little impact of DCFC on capacity loss in either Seattle or Phoenix; however, in Phoenix we see a significant impact of BTMS on capacity.
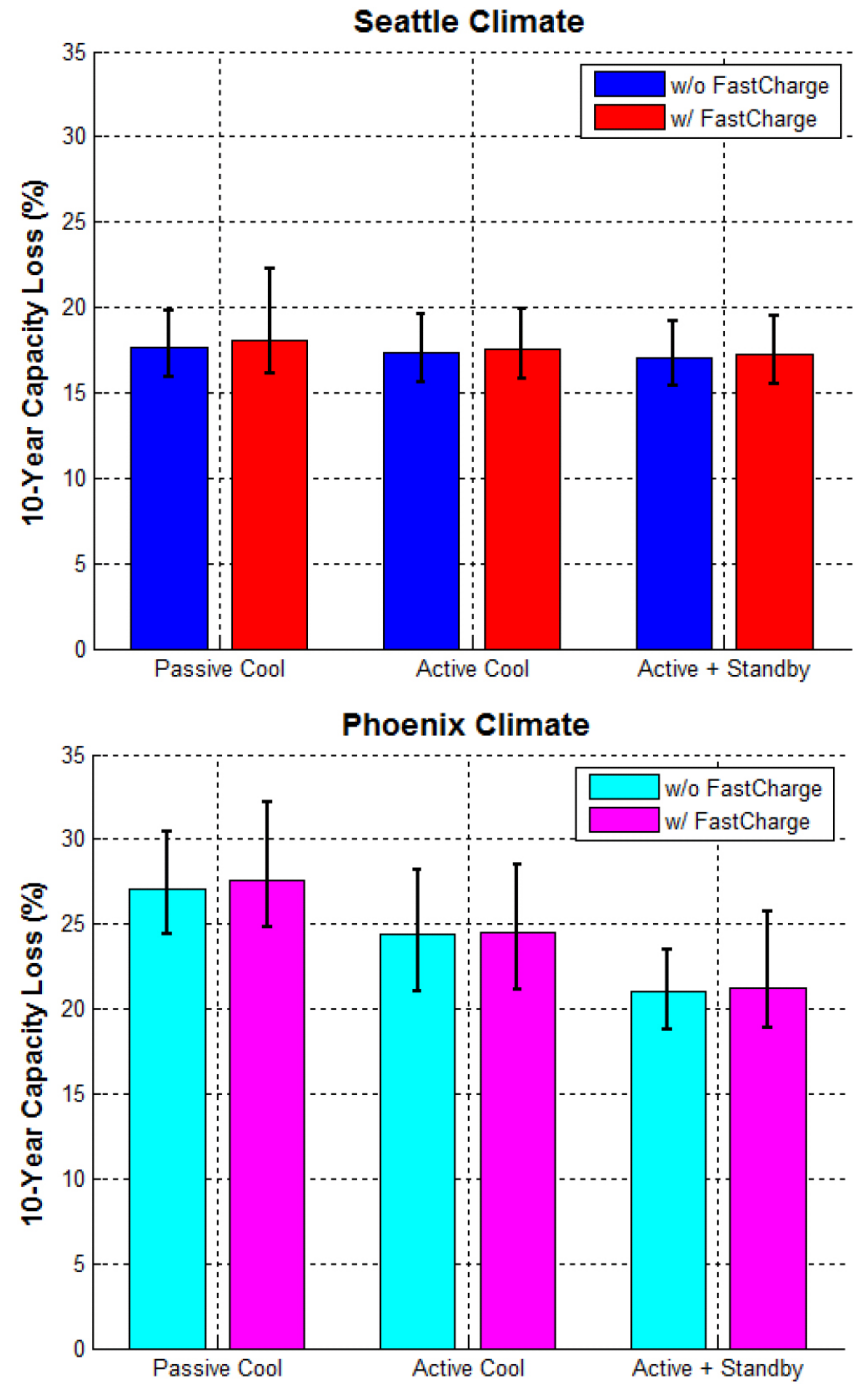

Figure 11. Effect of DCFCs and BTMSs on battery capacity loss in Seattle (top) and Phoenix (bottom)

While the nearly negligible impact of DCFC usage on battery capacity fade may be surprising to some, it is important to point out that DCFCs are used quite sparingly in our driver histories. Most drivers use a DCFC less than once per month (Figure 6), and when they are utilized, they typically charge the battery less than $60 \%$ (Figure 9). Further, recent tests where DCFCs are used twice per day to charge Nissan LEAFs driving in Phoenix have shown that the difference in capacity loss due to fast charger use (as compared to an otherwise identical case using Level 2 charging) after 50,000 miles of driving is less than $3 \%[\underline{31}]$. Thus, our results employing more realistic, less aggressive fast charging habits is to be expected. We do note, however, that it is possible that alternative battery chemistries not addressed herein could sustain considerable losses in capacity or increases in resistance due to such low frequency DCFC usage (e.g., via particle fracture). Clearly, it would not be advisable to recommend fast charging such chemistries, and thus analysis of such cases is not addressed herein.
Where the effect of DCFC use is most noticeable is in the maximum achieved battery temperature. As shown in Figure 12, comparison of cases with and without DCFC availability shows that maximum battery temperatures are $\sim 15^{\circ} \mathrm{C}$ higher for the median driver when fast charging is employed with a passive BTMS. In the presence of fast charging, our simulated maximum battery temperatures regularly exceed $45^{\circ} \mathrm{C}$ in Seattle and $60^{\circ} \mathrm{C}$ in Phoenix, so high that they could pose a safety risk if charging and/or driving is not impeded by onboard vehicle control systems. The addition of active battery cooling, however, can significantly moderate maximum battery temperatures, especially when employed both while driving and charging.
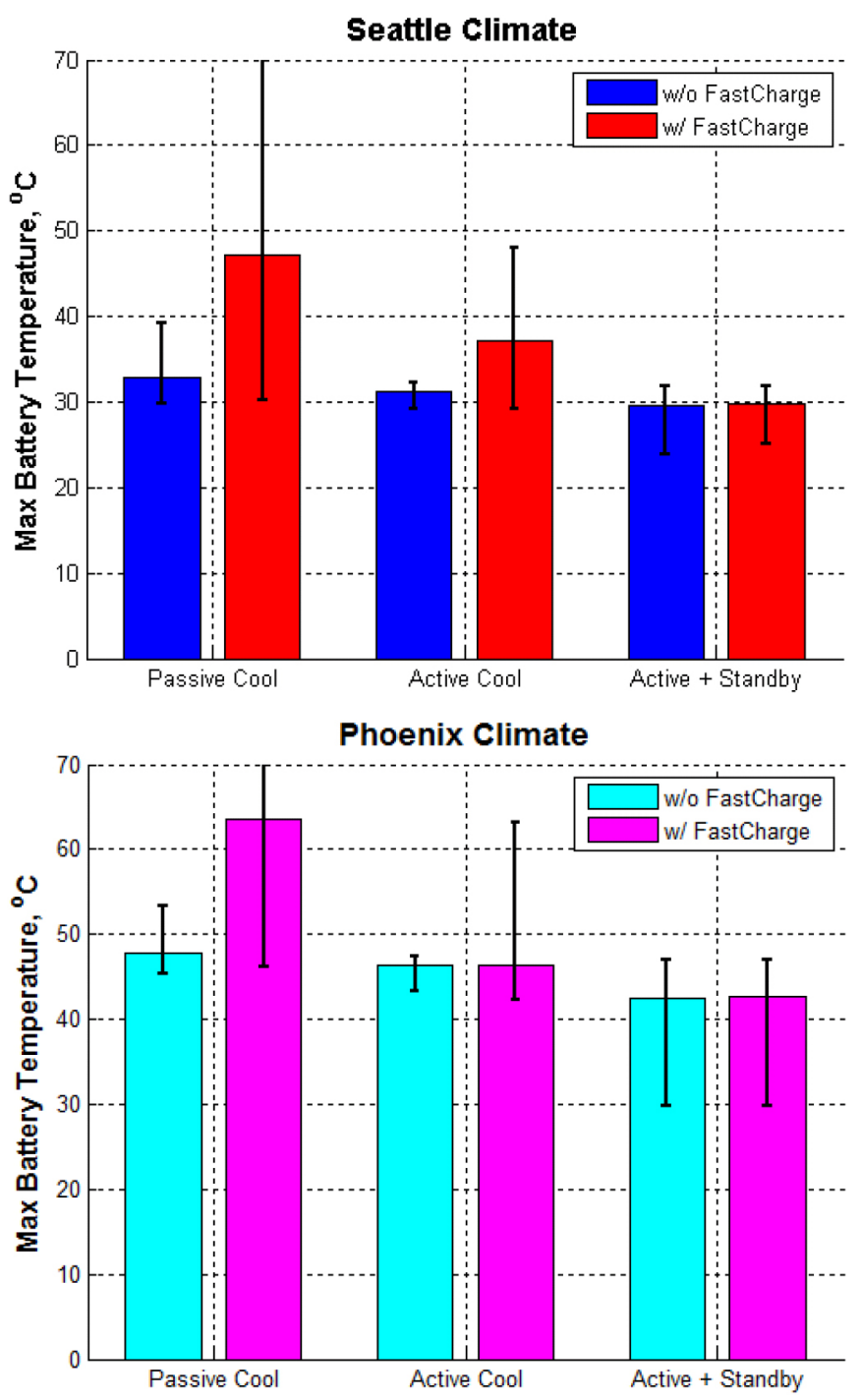

Figure 12. Effect of DCFCs and BTMS on maximum battery temperature in Seattle (top) and Phoenix (bottom)

\section{Results: Multi-Node Model}

To look more closely at the impact of DCFC on maximum battery temperature, we upgraded our battery model to the multi-node model. We restricted our study to the Phoenix climate on the basis that automotive manufacturers will need to design their vehicles to the worst-case environment. While we simulated all 180 travel histories, 

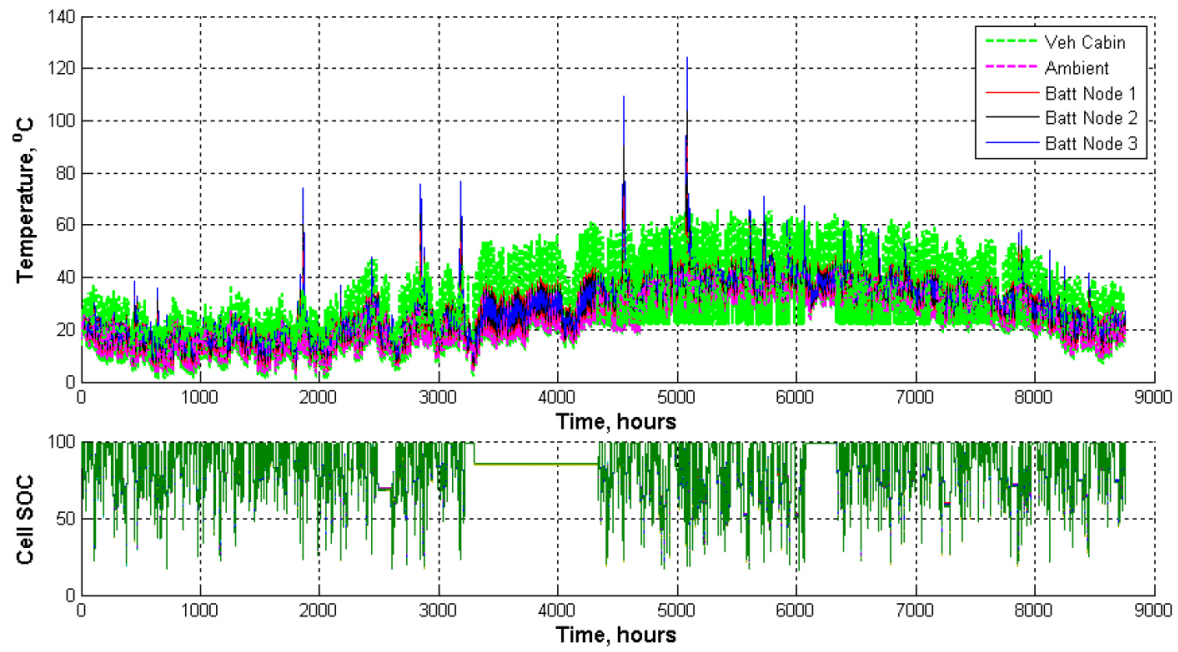

Figure 13. Single vehicle thermal response in Phoenix with passive BTMS

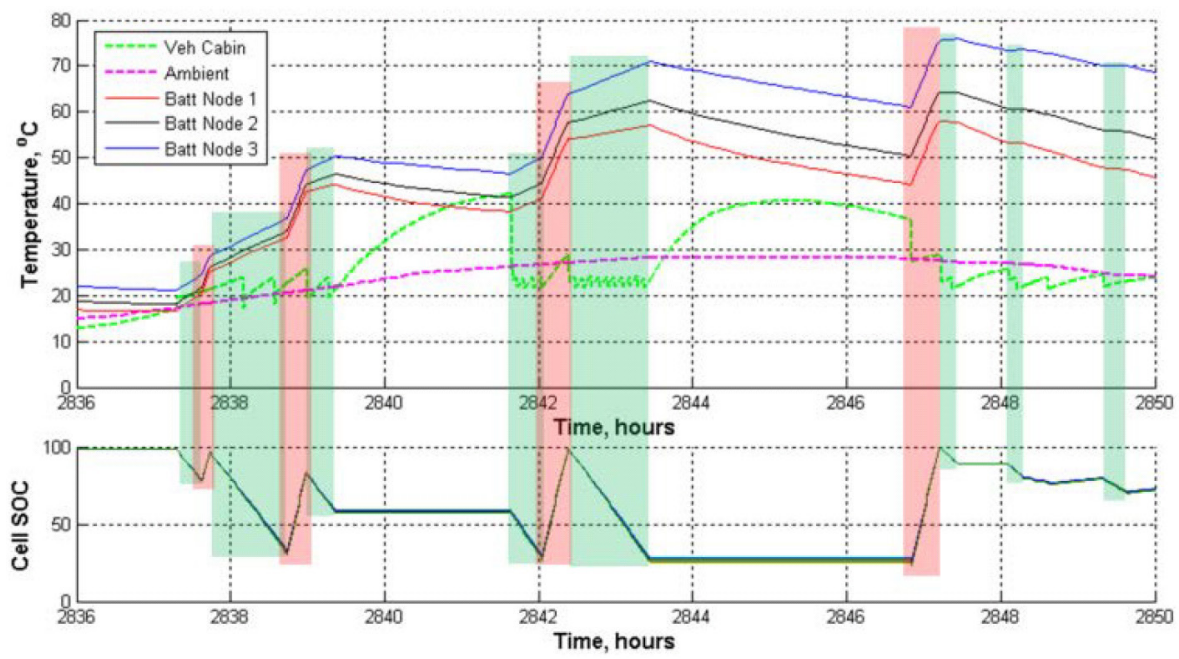

Figure 14. Single vehicle thermal response in Phoenix with passive BTMS (close-up); transparent green and red tiles overlaid on driving and DCFC events, respectively

we began with the presentation of a single vehicle to illustrate how fast charger use affects maximum battery temperatures, as shown in Figure 13. Note that there are five occasions over the course of the year at which the simulated battery temperature exceeds $70^{\circ} \mathrm{C}$, and two that exceed $100^{\circ} \mathrm{C}$.

When viewing these results, it is important to recognize that BLAST-V is calibrated to simulate nominal thermal response. In cases such as these where battery temperature becomes excessive, the accuracy of the thermal model is likely to suffer. Further, battery degradation and abuse response at such high temperatures are not accounted for. Thus, the primary value of these simulations is to conclude that reaching such temperatures is possible and must be controlled, not to precisely quantify the effects of such conditions (we assume they are unacceptable).

Zooming in on one of these events (Figure 14) illustrates the cause of such high temperature excursions: repeated back-to-back drive and charge events with short or no rests in between. Not only does the presence of fast charging allow vehicles to travel further more continuously than is otherwise possible in its absence, but the fast charging itself is shown to elevate battery temperature at a faster rate than driving (compare, for example, an estimated battery discharge power of $18 \mathrm{~kW}$ when driving at $300 \mathrm{Wh} / \mathrm{mi}$ and $60 \mathrm{mi} / \mathrm{hr}$ to a fast charge rate of $50 \mathrm{~kW})$. Thus, it is not unreasonable that a battery and BTMS designed for use without fast charging could overheat when this option is presented.

In Figure 15, we present the data for the same tour but with a BTMS with active cooling operated in both driving and standby modes. The effect on battery temperature is immediately apparent: the maximum battery temperature is constrained to less than $30^{\circ} \mathrm{C}$.

Finally, we explore the variation in cell state of health within packs following 10 years of automotive service. Here we employ the multi-cell model only with the active BTMS operated during driving and standby, having recognized its necessity in the preceding investigation. Results for maximum thermal gradients presented in Figure 16 show that such gradients regularly exceed $11^{\circ} \mathrm{C}$ in the presence of fast charging. Such gradients, if sustained for long periods of time, would be expected to create large variations in cell state of health within a pack, which would then limit the utility of the vehicle.

The results for capacity fade and resistance growth are presented in $\underline{\text { Figures } 17}$ and $\underline{18}$, respectively. In each plot, an individual red and blue box plot is given for each travel history providing the maximum, 75 th percentile, 50 th percentile (median), 25 th percentile, and 

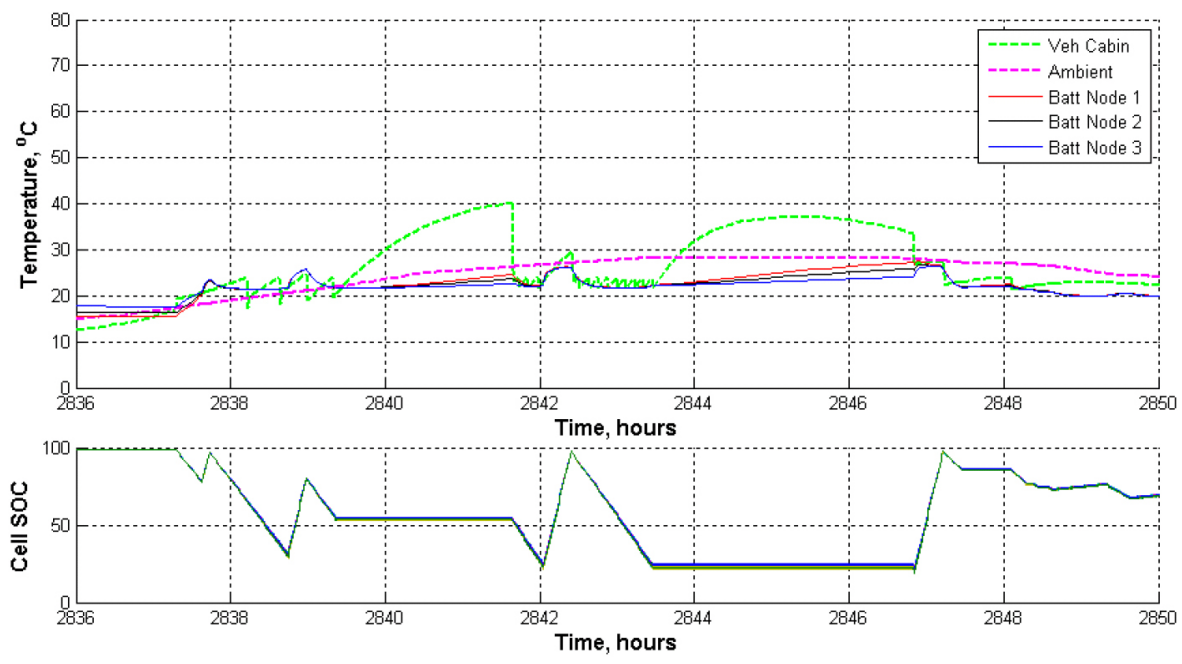

Figure 15. Single vehicle thermal response in Phoenix, AZ with active BTMS during driving and standby (close-up)

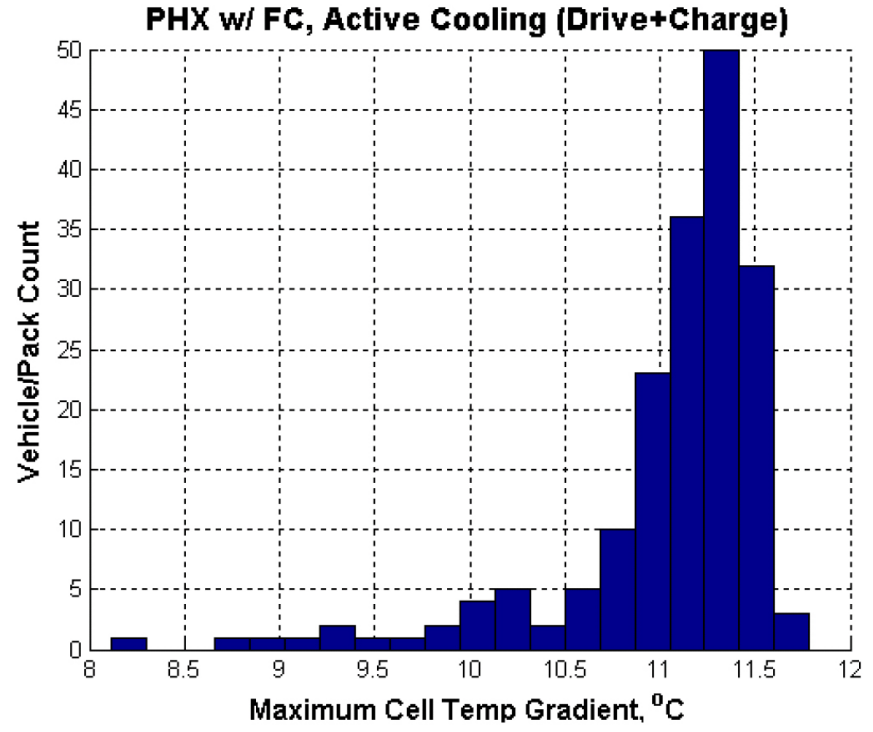

Figure 16. Maximum battery thermal gradients

Phoenix w/ FC, Active Cooling (Drive+Charge)

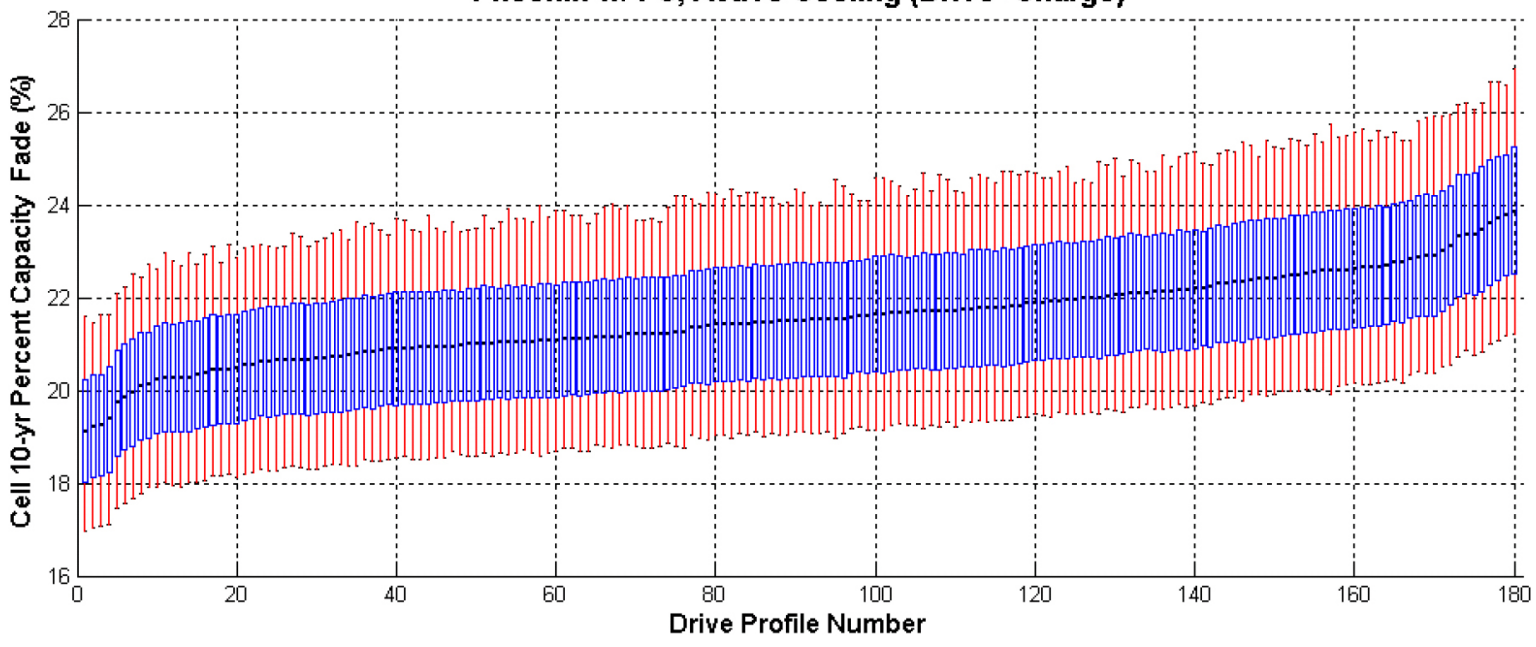

Figure 17. Capacity loss and variation within packs

minimum capacity fade (or resistance growth) of cells within the pack. The red whiskers represent the maximum and minimum. From these results we see that the amount of variation (either capacity fade or resistance growth) within each pack is fairly consistent across drive patterns at $\pm 2.5 \%$ capacity fade and $\pm 3.5 \%$ resistance growth.
This would appear to indicate that the maximum thermal gradients in Figure 16 are sufficiently temporary in duration as to have minimal effect on heterogeneous battery wear. However, the total amount of capacity fade and resistance growth can vary considerably across different drive patterns (resistance much more so than capacity). 


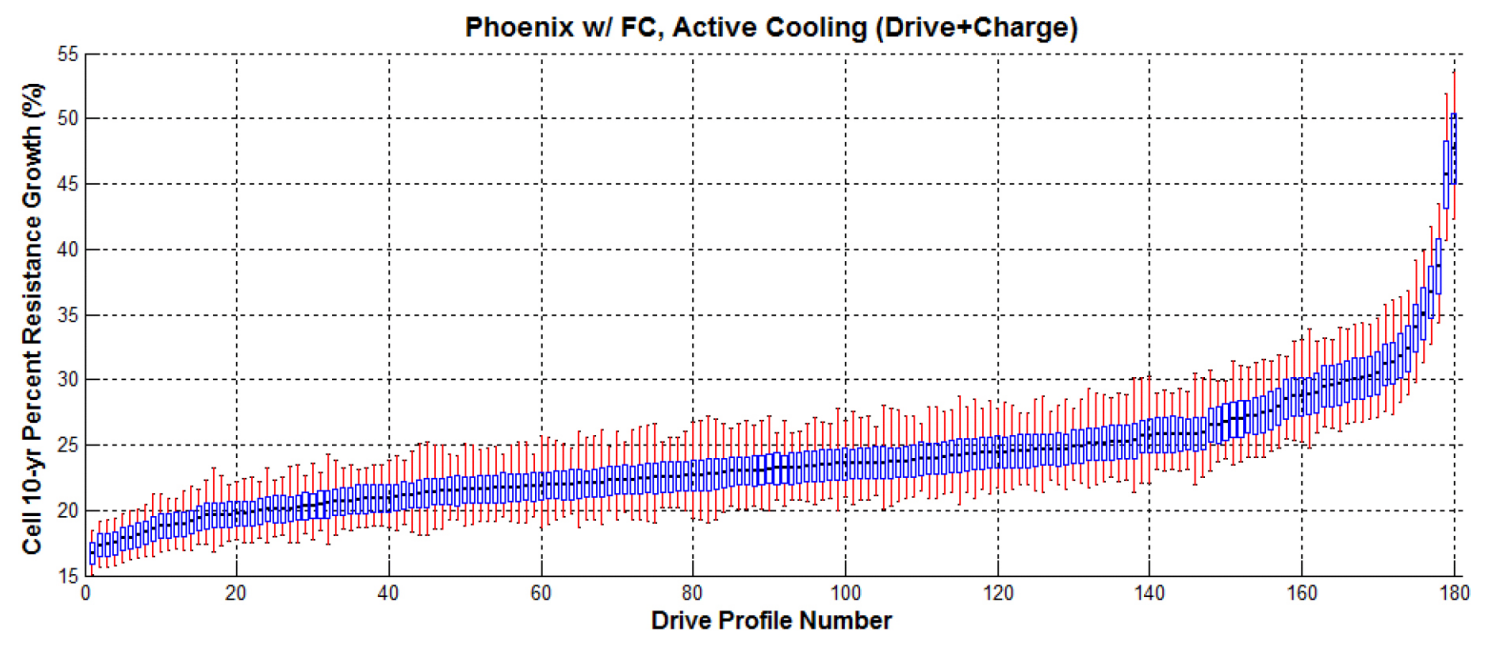

Figure 18. Resistance growth and variation within packs

\section{Conclusions}

In our study of the impact of realistic fast charging on simulated battery electrical, thermal, and degradation response we found that battery degradation is minimally affected for most drivers due to the low frequency of use. The largest challenge presented by fast charging to the battery is its effect on maximum battery temperature. In the presence of a passive BTMS, maximum battery temperatures can exceed safe operating limits due to repeated drive-charge sequences with short or no rests in between. However, this can be controlled by using BTMSs with active cooling capabilities or onboard vehicle controllers limiting charging and driving activities. The prior option is expected to be much more attractive in terms of driver satisfaction.

\section{References}

1. Anseán, D., González, M., Viera, J.C., García, V.M., Blanco, C., and Valledor, M., "Fast Charging Technique for High Power Lithium Iron Phosphate Batteries: A Cycle Life Analysis," J. Power Sources 239:9-15, 2013. Accessed January 26, 2015: http://www.sciencedirect.com/science/article/pii/ $\underline{\mathrm{S} 0378775313004357 .}$.

2. Chandrasekaran, R., "Quantification of Bottlenecks to Fast Charging of Lithium-Ion-Insertion Cells For Electric Vehicles," J. Power Sources 271:622-632, 2014. Accessed January 26, 2015: http://www.sciencedirect.com/science/article/pii/ $\underline{\mathrm{S} 0378775314011604}$.

3. Zhao, K., Pharr, M., Vlassak, J.J., and Suo, Z., "Fracture of Electrodes in Lithium-Ion Batteries Caused by Fast Charging," J. Appl. Phys. 108:073517, 2010. Accessed January 26, 2015: http://dx.doi.org/10.1063/1.3492617.

4. Patel, D.D., Tredeau, F.P., and Salameh, Z.M., "Temperature Effects on Fast Charging Large Format Prismatic Lithium Iron Phosphate Cells," accessed January 26, 2015: http://ieeexplore. ieee.org/stamp/stamp.jsp?tp=\&arnumber=5729073.

5. Bandyopadhyay, A., Wang, L., Devabhaktuni, V.K., Yang, R., and Green II, R.C., "Assessing the Effect of Fast Charging on the Battery Health of Plug-in Hybrid Electric Vehicles," accessed January 26, 2015: http://ieeexplore.ieee.org/stamp/ stamp.jsp?tp=\&arnumber $=6345617$.
6. Yan, J., Xu, G., Qian, H., and Xu, Y., "Battery Fast Charging Strategy Based on Model Predictive Control," accessed January 26, 2015: http://ieeexplore.ieee.org/stamp/stamp. jsp? $\mathrm{tp}=$ \&arnumber $=5594382$.

7. Phonrattanasak, P., and Leeprechanon, N., "Optimal Placement of EV Fast Charging Stations Considering the Impact on Electrical Distribution and Traffic Condition," International Conference and Utility Exhibition 2014 on Green Energy for Sustainable Development (ICUE 2014), Pattaya City, Thailand, 19-21 March 2014. Accessed January 26, 2015: http:// ieexplore.ieee.org/stamp/stamp.jsp?tp=\&arnumber=6828981.

8. Dharmakeerthi, C.H., Mithulananthan, N., and Saha, T.K., "Modeling and Planning of EV Fast Charging Station in Power Grid," accessed January 26, 2015: http://ieeexplore.ieee.org/ $\underline{\text { stamp/stamp.jsp?tp=\&arnumber }=6345008 \text {. }}$.

9. Cruz-Zambrano, M., Corchero, C., Igualada-Gonzalez, L., and Bernardo, V., "Optimal Location of Fast Charging Stations in Barcelona: A Flow-Capturing Approach," accessed January 26, 2015: http://ieeexplore.ieee.org/stamp/stamp. jsp?tp=\&arnumber $=6607414$.

10. Neubauer, J., and Wood, E., "The Impact of Range Anxiety and Home, Workplace, and Public Charging Infrastructure on Simulated Battery Electric Vehicle Lifetime Utility," J. Power Sources 257:12-20, 2014. Accessed January 26, 2015: http://www.sciencedirect.com/science/article/pii/ $\underline{\mathrm{S} 0378775314000998 .}$.

11. Neubauer, J., and Wood, E., "Thru-life Impacts of Driver Aggression, Climate, Cabin Thermal Management, and Battery Thermal Management on Battery Electric Vehicle Utility," J. Power Sources 259:262-275, 2014. Accessed January 26, 2015: http://www.sciencedirect.com/science/article/pii/ $\underline{\mathrm{S} 0378775314002766 .}$.

12. Wang, J., Liu, P., Hicks-Garner, J., Sherman, E., et al., "Cycle-Life Model for Graphite-LiFePO4 cells," J. Power Sources 196(8):3942-3948, 2011. Accessed December 12, 2014: http://www.sciencedirect.com/science/article/pii/ S0378775310021269.

13. Peterson, S. B., Apt, J., and Whitacre, J. F., "Lithium-Ion Battery Cell Degradation Resulting from Realistic Vehicle and Vehicle-to-Grid Utilization," J. Power Sources 195(8):23852392, 2010. Accessed December 12, 2014: http://www. sciencedirect.com/science/article/pii/S0378775309017443. 
14. Schmalstieg, J., Kabitz, S., Ecker, M., and Sauer, D.U., "AHolistic Aging Model for Li(NiMnCo)O ${ }_{2}$ Based 18650 Lithium-Ion Batteries,” J. Power Sources 257:325-334, 2014. Accessed December 12, 2014: http://www.sciencedirect.com/ science/article/pii/S0378775314001876.

15. Christensen, J., and Newman, J., "A Mathematical Model for the Lithium-Ion Negative Electrode Solid Electrolyte Interphase," $J$. Echem. Soc. 151(11):A1977-A1988, 2014. Accessed December 12, 2014: http://jes.ecsdl.org/content/151/11/A1977.full.

16. Ploehn, H. J., Premanand, R., and White, R. E., "Solvent Diffusion Model for Aging of Lithium-Ion Battery Cells," J. Echem. Soc. 151(3):A456-A462, 2004. Accessed December 12, 2014: http://www.che.sc.edu/faculty/white/2004solvent $\% 20$ diffusion $\% 20 \mathrm{hp} \% 20$ published.pdf.

17. Safari, M., and Delacourt, C., "Simulation-Based Analysis of Aging Phenomena in a Commercial Graphite/LiFePO4 Cell," J. Echem. Soc. 158(12):A1436-A1447, 2011. Accessed December 12, 2014: http://jes.ecsdl.org/content/158/12/A1436.full.pdf.

18. Smith, K., Earleywine, M., Wood, E., Neubauer, J. et al., "Comparison of Plug-In Hybrid Electric Vehicle Battery Life Across Geographies and Drive Cycles," SAE Technical Paper 2012-01-0666, 2012, doi: $10.4271 / 2012-01-0666$.

19. Wood, E., Neubauer, J., and Burton, E., "Quantifying the Effect of Fast Charger Deployments on Electric Vehicle Utility and Travel Patterns via Advanced Simulation," SAE Technical Paper 2015-01-1687, 2015, doi:10.4271/2015-01-1687.

20. Traffic Choices Study - Summary Report. Seattle, WA: Puget Sound Regional Council, April 2008.

21. National Renewable Energy Laboratory, National Solar Radiation Database, Typical Meteorological Year Database 3, Golden, CO, accessed July 2011. http://rredc.nrel.gov/solar/ old_data/nsrdb/1991-2005/tmy3/.

22. National Renewable Energy Laboratory, "Future Automotive Systems Technology Simulator," accessed October 31 2014, www.nrel.gov/fastsim.

23. "Revisions and Additions to Motor Vehicle Fuel Economy Label," Government Printing Office. Federal Register Vol. 76, No. 129, July 6, 2011. Accessed November 12, 2013: http:// www.gpo.gov/fdsys/pkg/FR-2011-07-06/html/2011-14291.htm.

24. Neubauer, J. and Wood, E., “Accounting for the Variation of Driver Aggression in the Simulation of Conventional and Advanced Vehicles," SAE Technical Paper 2013-01-1453, 2013, doi: $10.4271 / 2013-01-1453$.

25. Proprietary data provided by an OEM that wishes to remain anonymous.
26. U.S. Department of Energy, Alternative Fuels Data Center, “Alternative Fueling Station Locator," accessed January 29, 2014. http://www.afdc.energy.gov/locator/stations/.

27. Google Developers, Google Maps API Web Services "The Google Directions API," accessed October 31, 2014. https:// developers.google.com/maps/documentation/directions/.

28. The EV Project, accessed October 31, 2014. http://www. theevproject.com/.

29. Smart, J., "Latest Insights from The EV Project and ChargePoint America PEV Infrastructure Demos," GITT meeting at INL, August 12, 2014. Accessed January 26, 2015: http://avt.inel.gov/ pdf/EVProj/GITTEVProjectAug2014.pdf.

30. Beauregard, G., "Lessons Learned on the EV Project and DC Fast Charging," Navigant Research Webinar on Fast DC Charging for Electric Vehicles, April 9, 2013. Accessed January 26, 2015: http://www.theevproject.com/cms-assets/ documents/108217-328847.evp.pdf.

31. DC Fast Charge Effects on Battery Life and Performance Study - 50,000 Mile Update, accessed: December 23, 2014. http://avt. inl.gov/pdf/energystorage/DCFC Study_FactSheet_50k.pdf.

\section{Contact Information}

Jeremy Neubauer, D.Sc. Senior Engineer National Renewable Energy Laboratory jeremy.neubauer@nrel.gov

\section{Acknowledgments}

This study was supported by Dave Howell and Brian Cunningham of the Energy Storage, Vehicle Technologies Office, Office of Energy Efficiency and Renewable Energy, U.S. Department of Energy. Special thanks to Kandler Smith and Ahmad Pesaran of NREL.

\section{Definitions/Abbreviations}

BEV - battery electric vehicle

BEV75 - battery electric vehicle with 75-mile range

BLAST-V - Battery Lifetime Analysis and Simulation Tool for Vehicles

BTMS - Battery Thermal Management System

DCFC - direct current fast charger

HVAC - heating, ventilation, and air conditioning

kWh - kilowatt-hour(s)

NREL - National Renewable Energy Laboratory

SOC - state of charge

VMT - vehicle miles traveled

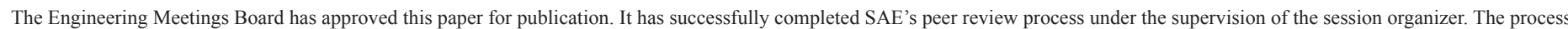
requires a minimum of three (3) reviews by industry experts.

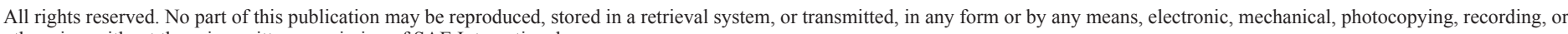
otherwise, without the prior written permission of SAE International.

Positions and opinions advanced in this paper are those of the author(s) and not necessarily those of SAE International. The author is solely responsible for the content of the paper.

ISSN 0148-7191

http://papers.sae.org/2015-01-1196 